\title{
An integrated MR/PET system: prospective applications
}

\author{
Heinz-Peter Schlemmer, ${ }^{1}$ Bernd J. Pichler, ${ }^{2}$ Robert Krieg, ${ }^{3}$ Wolf-Dieter Heiss ${ }^{4}$ \\ ${ }^{1}$ Department of Diagnostic and Interventional Radiology, University Hospital Tuebingen, Hoppe-Seyler-Strasse 3, 72076 Tueb- \\ ingen, Germany \\ ${ }^{2}$ Laboratory for Preclinical Imaging and Imaging Technology of the Werner Siemens-Foundation, Roentgenweg 13, 72076 \\ Tuebingen, Germany \\ ${ }^{3}$ Siemens AG, Healthcare, P.O. Box 3260, 91052 Erlangen, Germany \\ ${ }^{4}$ Max-Planck-Institut für Neurologische Forschung, Gleueler Str. 50, 50931 Cologne, Germany
}

\begin{abstract}
Radiology is strongly depending on medical imaging technology and consequently directing technological progress. A novel technology can only be established, however, if improved diagnostic accuracy influence on therapeutic management and/or overall reduced cost can be evidenced. It has been demonstrated recently that Magnetic Resonance Imaging (MRI) and Positron Emission Tomography (PET) can technologically be integrated into one single hybrid system. Some scientific arguments on the benefits are obvious, e.g., that simultaneous imaging of morphological and functional information will improve tissue characterization. However, crossfire of questions still remains: What unmet radiological needs are addressed by the novel system? What level of hardware integration is reasonable, or would software-based image co-registration be sufficient? Will MR/PET achieve higher diagnostic accuracy compared to separate imaging? What is the added value compared to other hybrid imaging modalities like PET/CT? And finally, is the system economically reasonable and has the potential to reduce overall costs for therapy planning and monitoring? This article tries to highlight some perspectives of applying an integrated MR/PET system for simultaneous morphologic and functional imaging.
\end{abstract}

Key words: Magnetic resonance imaging-MRI-Positron emission tomography-PET-MR-PET-Hybrid imaging

Correspondence to: Heinz-Peter Schlemmer; email: heinz-peter.schlemmer@med.uni-tuebingen.de
Combining two or more imaging modalities into one multi-modality machine is challenging. The imaging systems have to perform without mutual interferences while maintaining their full individual performance. Furthermore, the available space for accommodating the hardware from different scanners is often restricted. Another challenge is that the software components have to be integrated into one single analysis software package enabling and advanced workflow in routine radiology. A first realization of a multi-modality clinical imaging system is the combination of X-ray computed tomography (CT) and positron emission tomography (PET) [1]. While at the beginning, PET/CT was critically viewed and many skeptical clinicians asked about the diagnostic value of such a system, PET/CT is now fully accepted into the clinical diagnostic routine and has matured to an important tool in the field of clinical oncology [2].

\section{Instrumentation}

In PET/CT, the two scanners are hard-wired and implemented into a single gantry using one common bed, which is traveling through the two scanners. Thus, a full integration of PET and CT hardware, such as a common detector technology, is not realized yet. The highly different properties of low-energy X-rays with a high flux, as used in $\mathrm{CT}$, and the high-energy gamma rays in PET disable the use of a single detector to collect radiation from both imaging modalities. This technical limitation does not only result in increased costs to provide twice the imaging hardware, but does also disable simultaneous imaging. Thus, in PET/CT, the data are always acquired sequentially, first from $\mathrm{CT}$ and thereafter from PET. This limitation restricts the overlay accuracy of the two imaging data as well as the compensation of motion artifacts between the relatively slow PET acquisition and 
the fast CT data acquisition. However, a major advantage of $\mathrm{PET} / \mathrm{CT}$, besides providing multi-parametric morphological and functional information, is the feature to use the CT image for PET attenuation correction. In comparison to the conventional PET attenuation correction, where rotating ${ }^{68} \mathrm{G}_{\mathrm{e}}$ or ${ }^{137} \mathrm{C}_{\mathrm{s}}$ point or rod sources were used to acquire a transmission scan, using the CT information results in a reduction of scan time by nearly a factor of two.

Driven since more than 10 years, mainly by the small animal imaging research community, a new multimodality imaging device appears now on the horizon, which is the combination of PET with magnetic resonance imaging (MRI). Although to combine PET and MRI is more challenging than combining PET/CT, MR/ PET might compensate some of the shortcomings of $\mathrm{PET} / \mathrm{CT}$. This is feasible as the ultimate goal is to fully integrate the PET detector technology into an MRI gantry, enabling simultaneous PET and MRI data acquisition to provide spatial and temporal accurate correlated multi-parametric data. Simultaneous imaging not only improves the diagnostic value of MR/PET, but also enables respiratory or cardiac motion correction as well as track gut or patient movement.

Conventional PET detectors are based on relatively bulky photomultipliers (PMTs), which detect the scintillation light. PMTs are very sensitive even to weak magnetic fields and are therefore not suitable to be used in a combined MR/PET scanner. In addition, its size would steal too much space from the open magnet bore if integrated within a MRI scanner. As an alternative, new semiconductor-based light detectors, such as avalanche photodiodes (APDs) are replacing PMTs in combined $\mathrm{MR} / \mathrm{PET}$.

Initial animal MR/PET scanners [3-5] and very recently a first human brain MR-PET [6] are based on this new PET detector technology.

A high-resolution PET scanner prototype for brain imaging entitled BrainPET ${ }^{1}$, which is insensitive to magnetic fields, is built to slip-fit into a 3 Tesla whole MRI bore. This is the first human MR/PET scanner built by a commercial supplier. Since the intention of this MR/ PET scanner is a feasibility study to explore future MRPET applications, it already enables initial studies with patients. Since this prototype PET scanner is realized as an insert, it can be removed from the MRI scanner so that the 3 Tesla system can be used as standard imaging device. Although this prototype MR-PET allows only brain imaging with a standard MR bird cage coil, it provides all imaging capabilities as a future fully integrated whole-body MR/PET. Most importantly the PET and MRI data can be acquired simultaneously allowing not only a high-spatial overlay accuracy, but also an

\footnotetext{
${ }^{1}$ BrainPET is a Works in Progress device.
}

excellent temporal co-registration. This is in sharp contrast to PET/CT and might enable MR-based PET image motion correction for accurate abdominal or cardiac imaging. Considering all the advantages of simultaneous multi-parametric imaging and the much longer MR scan time, compared to CT, a sequential MR/PET device might not be very useful clinically.

The BrainPET uses PET block detectors-based APDs to detect the scintillation light from the lutetium oxyorthosilicate (LSO) crystals. The prototype camera achieves a $192 \mathrm{~mm}$ axial field of view (FOV) and 355-mm transaxial FOV of the PET scanner. The PET reveals a spatial resolution of about $2.5-3 \mathrm{~mm}$ in the reconstructed image, which is superior to other clinical PET systems. Evaluation studies have shown, that both systems, the PET and MRI, maintain their full individual imaging performance with minimal mutual interference between the two scanners. The MR scanner even allows the application of demanding sequences such as spectroscopy (MRS) or echo planar imaging (EPI), which is used for functional MRI (fMRI). First applications of the prototype demonstrated high resolution in phantom studies and the feasibility of simultaneous MRI and PET data acquisition in patients [6]. The image quality obtained with simultaneous MR/PET is comparable to stand-alone systems and allowed accurate co-registration of morphologic and functional information (Fig. 1).

\section{Co-registration vs. combination of $M R I$ and PET}

The combination of anatomical and functional information into one single image has been pursued for a long time, and various strategies have been applied for coregistration of PET or SPECT and MRI or CT images [1, 7]. On principle, PET quantification in the picomolar range of various functional parameters (e.g., metabolism, flow, receptor-ligand binding, enzyme activity, gene expression) can be correctly correlated to normal and pathologically altered anatomy, and additionally complemented with dynamic information from functional MR. Combining anatomic, functional, and metabolic information of both modalities permits the complementary assessment of biological properties (Table 1).

Software fusion of PET and CT or MRI images suffer from limitations: the imaging modalities usually are located in different laboratories, the image properties, e.g., resolutions are different and suffer from distortion, image translations and rotations, partial volume effects cannot be compared, and dynamic changes cannot be followed in an identified structure. The fusions of brain images - which will be the main objective of this discussion - is relatively easy owing to its rigid structure, whereas especially in the abdomen or thorax an exact relocation of the patient is difficult and makes the alignment of images from different modalities uncertain. A 

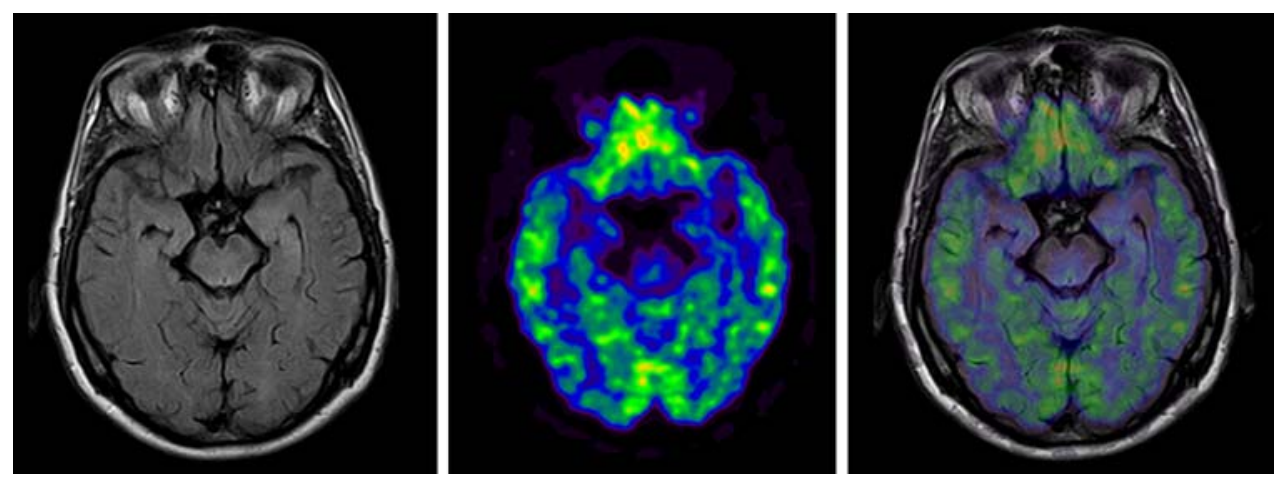

Fig. 1. MR (FLAIR sequence), $\left[{ }^{18} \mathrm{~F}\right] \mathrm{FDG}$ PET and fused images of the brain acquired simultaneously with an integrated MR/PET system. Acquisition time $15 \mathrm{~min}$.

Table 1. Assessment of biological properties by MR and PET

\begin{tabular}{l} 
MR \\
\hline Morphology \\
Water diffusion capacity (DWI) \\
Vascular anatomy (MRA) \\
Perfusion (PWI, DCE-MRI) \\
Tissue metabolites (MRS) \\
Functional activation (fMRI) \\
Cerebral fiber tracts (DTI) \\
Oxygen consumption ( $\left.{ }^{17} \mathrm{O}\right)$ \\
Migration of cells (Fe labeling)
\end{tabular}

PET

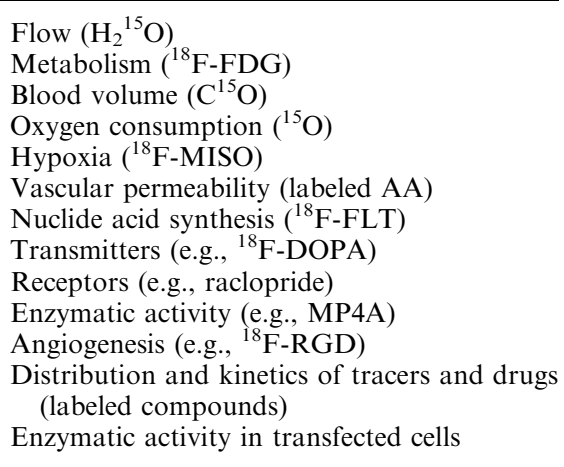

combined multi-modal imaging device ensures a reliable alignment of images and permits the combination of morphology with multi-functional information from PET, MRI, fMRI, and MRS. The first simultaneous MR/PET study demonstrates the feasibility of this approach and may justify some speculations on further applications [6].

\section{Potential applications of $M R / P E T$ in the brain}

Integrated MR-PET is on the verge of being applied to clinical neurosciences as it is already utilized in preclinical research [4]. The combined system will extend the impact of simultaneous imaging of morphology and function as well as metabolism. Co-registration of MR and PET images was successfully applied for detection and staging of gliomas $[8,9]$ as well as for identification of eloquent areas in the vicinity of tumors, which is important for planning surgery [10]. Combined PET and MRI information has gained a place in early diagnosis of dementia and mild cognitive impairment [11] and of degenerative disorders, e.g., cerebellar atrophy, Huntington chorea. It was of special clinical value in the detection of epileptic foci accessible for surgery [12] and in the identification of metabolic activity, transmitter concentration and enzyme expression in small brain structures [13]. In experimental focal brain ischemia and ischemic stroke, co-registration of regional values for cerebral blood flow, oxygen utilization and glucose metabolism to early and late MRI permitted the distinction of irreversibly damaged and functionally impaired, but morphologically preserved, i.e., penumbra tissue in the acute phase, a differentiation valid for the selection of appropriate treatment strategies [14].

These future applications of combined MR/PET will gain new dimensions with the simultaneous MR and PET procedures becoming possible with BrainPET. Simultaneous data acquisition will allow the addition of kinetic, functional, and metabolic information for real time multi-parametric functional imaging:

- MRA will add the vascular origin of changes in cerebral blood flow, oxygen consumption and metabolism in vascular diseases, including subarachnoid hemorrhages.

- MRS will add additional metabolic information, e.g., lactate concentration, ATP, choline, N-acetyl-aspartate, to regional metabolic data from PET $\left({ }^{15} \mathrm{O}_{2}\right.$, FDG, FLT) in stroke, gliomas, and degenerative disorders.

- Perfusion (PWI) can be related to hypoxic markers $\left({ }^{18} \mathrm{~F}\right.$-MISO PET) and metabolic markers as lactate, choline, N-acetyl-aspartate (MRS) in gliomas and ischemia.

- DWI-PWI can be performed during PET measurements of ${ }^{15} \mathrm{O}_{2}$ and $\mathrm{H}_{2}{ }^{15} \mathrm{O}$ for differentiation of 
intravascular perfusion, tissue blood flow, penumbra, and irreversible tissue damage in ischemic stroke.

- Comparative activation studies with fMRI and $\mathrm{H}_{2}{ }^{15} \mathrm{O}$ (or FDG) PET will settle the discussion on the exact localization of activation patterns (intravascular vs. tissue signals).

- The effect of activation by specific tasks on transmitter release or receptor binding can be analyzed by combining fMRI with PET of, e.g., DOPA or raclopride.

- Effects of drugs and of withdrawal (e.g., nicotine) on task performance can be studied by fMRI and PET receptor ligands (e.g., ${ }^{11} \mathrm{C}$-raclopride).

The combination of MR and PET in the acquisition of dynamic data will improve modeling for quantification of metabolic values

- PWI could be used to determine flow-dependent kinetic constants for compartmental analysis of PET data (e.g., FDG, FLT).

- Perfusion can be assessed by PWI simultaneously with metabolic and functional parameters from PET.

- The dynamics of distribution and the kinetics of various tracers or drugs can be obtained in various brain structures.

- A new MR tracer for oxygen utilizations $\left({ }^{17} \mathrm{O}\right)$ could be validated on simultaneously recorded ${ }^{15} \mathrm{O}$ utilization by PET $\left(\mathrm{O}_{2} \rightarrow \mathrm{H}_{2} \mathrm{O}\right)$.

- Arterial spin labeling (ASL) MRI could be validated by $\mathrm{H}_{2}{ }^{15} \mathrm{O}$-PET for measurement of $\mathrm{CBF}$.

For complex studies of brain functions special combinations of modalities or additional investigative procedures may open new insights into the organization of the brain and the changes in disease:

- Diffusion tensor tracking can be added to activation studies and effects on transmitter release and receptor occupancy as well as on metabolism in connected areas can be analyzed as substrates of connectivity in networks.

- Repetitive transcranial magnetic stimulation can be used to activate or inhibit selected areas of the cortex, and the effect on metabolism and the involvement of transmitter systems can be analyzed.

- Deep brain stimulation is nowadays a means to ameliorate neurological symptoms and improve abnormal behavior [15]. The effect of this invasive manipulation on metabolism and transmitter/receptor interaction in defined regions together with the connecting fiber tracts can be evaluated.

An innovative experimental field which is opening up with this new combined imaging modality is molecular and cellular imaging.

- Angiogenesis is a fundamental process in various physiologic and pathologic processes. Visualization, quantification, and monitoring of angiogenesis are of interest in various fields, including oncology and cardiology. Molecules regulating angiogenesis include growth factor receptors and integrins. Cyclic RGD peptides bind to activated integrin $\alpha_{v} \beta_{3}$, and ${ }^{18} \mathrm{~F}$ galacto RGD [16] has been tested in humans with high uptake in highly vascularized tumors. As an alternative approach labeled ligands to the vascular endothelial growth factor receptor were applied. Combined with dynamic contrast-enhanced MRI-yielding vascular and extravascular volumes, vascular permeability and perfusion - angiogenesis PET-markers will permit the monitoring of angiogenetic and antiangiogenetic treatment [17].

- Targeted gene transfer by various vectors can be used to express foreign enzymes in cells; this strategy can be applied, e.g., to make malignant cells susceptible to specific drugs, which are toxic only to those cells expressing this enzyme. In an experimental glioma model the efficacy of this treatment strategy with transfer of Herpes virus thymidine kinase gene and application of gancyclovir was demonstrated; preliminary clinical tests also showed some efficacy of this treatment option [18]. The effect of this therapy can be followed by multitracer PET and verified on MRI.

- Implantation of embryonic stem cells into striatum lesioned by 6-OHDA injection in rats leads to proliferation and differentiation of dopaminergic cells. This could be demonstrated by co-registering MRI and PET of the specific dopamine transporter ligands ${ }^{11} \mathrm{C}$ 2betacarbometoxy-3beta-(4fluorophenyl)tropane

(CFT) where CFT binding was restored in the region of post-mortem documented $\mathrm{TH}$-immunoreactive neurons. The restored functional activity of these implanted cells was demonstrated by the response to amphetamine causing increase in $\mathrm{rCBV}$ due to $\mathrm{DA}$ release [19]. Simultaneous MR/PET can be used to show the viability and differentiation of transplanted cells and their effect on the neuronal networks.

- Cell replacement approaches are an innovative strategy for treatment of various neurological disorders. For the development of these approaches in animal models it is essential to monitor the location and follow the migration of grafted stem or progenitor cells. Various strategies have been proposed for labeling these cells with ultrasmall superparamagnetic iron oxide particles and micronsized iron oxide particles [20]. In focal experimental ischemia labeled stem cells migrated during 3 weeks from the contralateral implantation site along the corpus callosum to the ventricular walls and massively populated the border zone of the damaged brain tissue on the lesioned hemisphere. A combination of these MRI studies with PET procedures could demonstrate the viability of the cells as well as their integration into functional networks. Monitoring cell viability and migration by 
MRI combined with indicators of function from PET might become also a qualifying step in strategies relying on transplantation of fetal grafts, e.g., in Parkinson's disease.

\section{Comparison of $M R / P E T$ and PET/CT for body imaging}

Potential clinical applications of MR/PET in body imaging will include predominantly the fields of oncology and cardiovascular diseases.

Although morphologic imaging is important, the assessment of tissue aggressiveness or viability cannot be based on pathoanatomic information alone. The introduction of PET/CT has considerably improved oncologic imaging by combining anatomical with functional information concerning cell metabolism and receptor density. More sensitive and specific diagnosis could be achieved for different tumor entities enabling individualized therapy planning, monitoring and evaluation of recurrent disease. Current indications for PET/CT include, e.g., non-small cell lung cancer (NSCLC), esophageal cancer, colorectal cancer or lymphoma. As highresolution whole-body MRI has become feasible [21], clinical studies compared to whole-body CT and PET/ CT have shown complementary advantages of MR and PET [22, 23].

MR provides anatomical details with high soft tissue contrast and a variety of functional information about perfusion, diffusion, and metabolism. PET enables assessment of metabolic abnormalities in mass lesions and their changes early during therapy before changes in tumor size can be measured. Improved spatial, contrast, and temporal resolution of MR implies several advantages of simultaneous MR/PET as compared sequential $\mathrm{PET} / \mathrm{CT}$.

1. The superior soft tissue contrast of MR is in general relevant for all types of soft tissue tumors regarding detection, delineation, characterization, and staging. However, CT is still more sensitive than MR in revealing small lung nodules, bone and bone structures.

2. Dynamic MR studies can yield functional tissue parameters for quantitation of perfusion without additional radiation exposure, and PET studies can be simultaneously performed. The resulting images represent the tissue and the vascular component of the tumor.

3. The complementary morphological and metabolic data can be relevant for defining biopsy targets, particularly by separating areas of active tumor from inflammation, fibrosis or necrosis. For this, additional information obtained with MRS and/or special tracers, as ${ }^{11} \mathrm{C}$ - or ${ }^{18} \mathrm{~F}$-choline in prostate cancer, ${ }^{18} \mathrm{~F}$-L-thymidine in germ cell tumors and with
${ }^{11} \mathrm{C}$-methionine and ${ }^{18} \mathrm{~F}$-L-thymidine in brain tumors, or by imaging of angiogenesis $\left({ }^{18} \mathrm{~F}-\mathrm{RGD}\right)$.

4. Combined morphological and metabolic imaging is important for the evaluation of early treatment response. Metabolic information assessed by FDG, MET, or FLT is more sensitive to detect therapy-induced metabolic and necrotic tissue damage. Additional high-resolution morphologic information may be potentially helpful in the planning of subsequent surgery and radiotherapy.

5. Fast MR sequences can improve the image quality of PET by providing kinetic information of breathing or cardiac activity and thereby minimizing artifacts in PET images, e.g., by (respiratory and cardiac gating). Cardiac gating is of especial importance for imaging of the myocardium depicting pathological changes, e.g., infarcts and scars. Changes in blood flow and metabolism and possible neovascularization can be detected within the infarct zone by PET applying, e.g., ${ }^{18} \mathrm{~F}$ RGD as tracer for integrine activation. As a prerequisite, however, precise motion correction and exact morphologic-metabolic co-registration are important.

6. MRI causes no additional radiation exposure, which is of importance especially in dynamic contrast-enhanced studies and repeated examinations. This is of particular importance for follow-up of younger individuals after curative treatment, e.g., testicular tumors or malignant melanoma.

Regarding the screening for metastases, MR is superior in the detection of brain, liver, and bone marrow metastases and PET is important for characterizing lymph nodes and soft tissue masses [22, 24]. MRI provide more anatomical details than CT, e.g., in the head and neck region, where the differentiation of closed anatomical structures like mucosa, submucosal tissues, salivary glands, muscles, lymph nodes, and blood vessels is indispensible for making correct diagnoses and therapy plans. MR is in general preferable for abdominal and pelvic imaging. Detailed MR/PET information about anatomy and metabolism will enable better tumors staging of several tumor entities, e.g., liver, cervical, prostate, urinary bladder or rectal cancer. In thoracic tumors, however, PET/CT will still be preferable because $\mathrm{CT}$ is the imaging modality of choice for the examination of the lung.

Regarding the detection of bone metastases, FDG$\mathrm{PET} / \mathrm{CT}$ can be false negative in case of small metastases. Minor tracer uptake may not be visualized because of physiological FDG tracer uptake of normal bone marrow and CT may be negative as changes in bone architecture may not yet be present. MRI on the other hand images the bone marrow itself and has consequently been proven to be even more sensitive than conventional bone scintigraphy [25].

Another major diagnostic problem in oncology is the differentiation of therapy-related scar tissue from recur- 
rent tumor, e.g., in rectal carcinoma after surgery and/or radiochemotherapy. Suspicious tissue in the pelvis can be differentiated from normal anatomical structures, while FDG-PET enables information about their probable pathologic tracer uptake.

Non-oncologic applications of MR/PET include the assessment of cardiovascular diseases. High-resolution MRI and PET of the beating heart and pulsating arteries will be feasible. MR-information about cardiac and respiratory motion can be used for pro- or retrospective MR-gating of the PET data acquisition. Application of whole-body MR/PET in the field of cardiovascular imaging will include the evaluation of myocardial perfusion, viability plaque imaging and assessment of inflammatory disorders, e.g., aortitis. Whole-body MRAngiography can be combined with cardiac MR.

It is important to mention, however, that wholebody MR/PET applications will require a reliable method for MR-based photon attenuation correction, which is challenging and still under development until now $[26,27]$.

\section{Conclusions}

There is no doubt that PET/CT will remain an important diagnostic instrument in clinical routine, but further scientific and clinical progress can be anticipated by hybrid MR/PET imaging. The integrated MR/PET will definitely serve in research for the exploration of molecular mechanisms in healthy individuals as well as in complex disease. If it can be shown that a combined reading of PET and MRI improves diagnostic accuracy or efficacy of treatments via better monitoring, it may become part of medical guidelines. However, it needs to be proven that the integration of both modalities is economically superior to two individual systems.

The main economic argument for the integration is that PET and MR are both complex and time-consuming examinations and subsequent imaging is consequently cumbersome for the patient and laborious regarding the workflow. Thus the costs for two separate diagnoses in both modalities pose an immense burden to the patients and the healthcare providers. As true simultaneous PET and MR imaging become feasible, the overall scan time can significantly be reduced compared to sequential scanning. Simultaneous MR/ PET imaging will also cut down the time needed for visualization and interpretation of separate and/or fused images. These savings in cost support the aforementioned requirement from healthcare systems of a higher quality at lower cost and might open the path for MR/ PET into clinical routine.

Open Access. This article is distributed under the terms of the Creative Commons Attribution Noncommercial License which permits any noncommercial use, distribution, and reproduction in any medium, provided the original author(s) and source are credited.

\section{References}

1. Townsend DW (2001) A combined PET/CT scanner: the choices. J Nucl Med 42:533-534

2. Antoch G, Saoudi N, Kuehl H, et al. (2004) Accuracy of whole body dual modality fluorine-18-2-fluoro-2-deoxy-D-glucose positron emission tomography and computed tomography (FDG-PET/ CT) for tumor staging in solid tumors: comparison with $\mathrm{CT}$ and PET. J Clin Oncol 22(21):4357-4368

3. Catana C, Procissi D, Wu Y, et al. (2008) Simultaneous in vivo positron emission tomography and magnetic resonance imaging. Proc Natl Acad Sci U S A 105:3705-3710

4. Judenhofer MS, Wehrl HF, Newport DF, et al. (2008) Simultaneous PET-MRI: a new approach for functional and morphological imaging. Nat Med 14:459-465

5. Raylman RR, Majewski S, Velan SS, et al. (2007) Simultaneous acquisition of magnetic resonance spectroscopy (MRS) data and positron emission tomography (PET) images with a prototype MRcompatible, small animal PET imager. J Magn Reson 186:305-310

6. Schlemmer H-P, Pichler BJ, Schmand M, et al. (2008) Simultaneous MR/PET imaging of the human brain: a feasibility study. Radiolog 248(3):1028-1035

7. Vollmar S, Cizek J, Su M, et al. (2005) VINCI: visualization and fast co-registration of multi-modal imaging data of animal and human studies. Mol Imaging 4:267

8. Chen W (2007) Clinical applications of PET in brain tumors. J Nucl Med 48:1468-1481

9. Jacobs AH, Kracht LW, Gossmann A, et al. (2005) Imaging in neurooncology. NeuroRx 2:333-347

10. Thiel A, Habedank B, Herholz K, et al. (2006) From the left to the right: how the brain compensates progressive loss of language function. Brain Lang 98:57-65

11. Herholz K (2003) PET studies in dementia. Ann Nucl Med 17:7989

12. Koepp MJ (2005) Woermann FG. Imaging structure and function in refractory focal epilepsy. Lancet Neurol 4:42-53

13. Eggers C, Szelies B, Bauer B, et al. (2007) Imaging of acetylcholine esterase activity in brainstem nuclei involved in regulation of sleep and wakefulness. Eur J Neurol 14:690-693

14. Heiss WD (2000) Ischemic penumbra: evidence from functional imaging in man. J Cereb Blood Flow Metab 20:1276-1293

15. Sturm V, Lenartz D, Koulousakis A, et al. (2003) The nucleus accumbens: a target for deep brain stimulation in obsessive-compulsive- and anxiety-disorders. J Chem Neuroanat 26:293-299

16. Beer AJ, Grosu AL, Carlsen J, et al. (2007) [18F]galacto-RGD positron emission tomography for imaging of alphavbeta3 expression on the neovasculature in patients with squamous cell carcinoma of the head and neck. Clin Cancer Res 13(22 Pt 1):6610-6616

17. Hsu AR, Chen X (2008) Advances in anatomic, functional, and molecular imaging of angiogenesis. J Nucl Med 49:511-514

18. Jacobs AH, Rueger MA, Winkeler A, et al. (2007) Imaging-guided gene therapy of experimental gliomas. Cancer Res 67:1706-1715

19. Bjorklund LM, Sanchez-Pernaute R, Chung S, et al. (2002) Embryonic stem cells develop into functional dopaminergic neurons after transplantation in a Parkinson rat model. Proc Natl Acad Sci U S A 99:2344-2349

20. Hoehn M, Himmelreich U, Kruttwig K, et al. (2008) Molecular and cellular MR imaging: potentials and challenges for neurological applications. J Magn Reson Imaging 27(5):941-954

21. Schlemmer HP, Schäfer J, Pfannenberg C, et al. (2005) Fast wholebody assessment of metastatic disease using a novel magnetic resonance imaging system. Invest Radiol 40(2):64-71

22. Pfannenberg C, Aschoff P, Schanz S, et al. (2007) Prospective comparison of $18 \mathrm{~F}$-fluorodeoxyglucose positron emission tomography/computed tomography and whole-body magnetic resonance imaging in staging of advanced malignant melanoma. Eur J Cancer $43: 557-564$

23. Schmidt GP, Kramer H, Reiser MF, et al. (2007) Whole-body magnetic resonance imaging and positron emission tomographycomputed tomography in oncology. Top Magn Reson Imaging 18(3):193-202

24. Muller-Horvat C, Radny P, Eigentler TK, et al. (2006) Prospective comparison of the impact on treatment decisions of whole-body magnetic resonance imaging and computed tomography in patients with metastatic malignant melanoma. Eur J Cancer 42:342-350 
25. Ghanem N, Uhl M, Brink I, et al. (2005) Diagnostic value of MRI in comparison to scintigraphy, PET MS-CT and PET/CT for the detection of metastases in bone. Eur J Radiol 55(1):41-55

26. Beyer T, Weigert M, Quick HH, et al. (2008) MR-based attenuation correction for torso-PET/MR imaging: pitfalls in mapping MR to CT data. Eur J Nucl Med Mol Imaging 35(6):1142-1146
27. Hofmann M, Steinke F, Scheel V, et al. (2008) MR-based attenuation correction for PET/MR: a novel approach combining pattern recognition and Atlas registration. $\mathbf{J}$ Nucl Med, in press 